\title{
Review of: "Integrated analysis reveals FOXA1 and Ku70/Ku80 as direct targets of ivermectin in prostate cancer"
}

jing jing

Potential competing interests: The author(s) declared that no potential competing interests exist.

This manuscript described a really good study on the pharmacological mechanism of ivermectin in prostate cancer. However, I have two suggestions. First, are the non-tumorigenic human prostate primary stromal cells from patients with BPH AR-positive or AR-negative? It should be used as a non-cancer cell control when investigating the AR-dependent or AR-independent effects of ivermectin in prostate cancer cells. Second, Ku70 protein has been reported to regulate cell apoptosis by the Bax signalling and deubiquitination of $\mathrm{Mcl}-1$ protein. As the ivermectin was found to affect Ku70, these pathways involved in cell apoptosis regulation should also be investigated to explain the pharmacological mechanism of ivermectin. 